\title{
GENERATION OF X-RAY FLUCTUATIONS IN X-RAY BINARIES
}

\author{
S. MINESHIGE ${ }^{1}$, M. TAKEUCHI ${ }^{1}$, H. NEGORO ${ }^{2}$ \\ 1. Department of Astronomy, Faculty of Science, \\ Kyoto University, Sakyo-ku, Kyoto 606-01, Japan \\ 2. Institute of Space and Astronautical Science, \\ Yoshinodai, Sagamihara 229, Japan
}

\begin{abstract}
Short-term aperiodic fluctuations with $1 / f$-like power spectral density (PSD), where $f$ is the frequency, are characteristics of X-ray radiation from X-ray binaries. To investigate a mechanism creating fluctuations, we construct a cellular-automaton model for accretion disks based on the concept of self organized criticality (SOC). In this model, mass accretion takes place either by an avalanche triggered when the local mass density exceeds some critical value, or by a gradual diffusion occurring regardless of the critical condition. With this model, we can reproduce the observational PSD, the distributions of peak intensities of X-ray shots, and non-random temporal distribution of the shots. Possible accretion disk models producing $1 / f$-like $\mathrm{X}$-ray fluctuations are discussed. We conclude that such disks are likely to be advection dominated.
\end{abstract}

\section{Introduction}

A long time has passed since the discovery of short-term X-ray variations from stellar black-hole candidates (see the review by Oda 1977). Through power spectrum analysis it has been claimed that such X-ray time variations are similar to $1 / f$ noise. A similar $1 / f$-like PSD was found also in neutron-star X-ray binaries in the low-luminosity state (Makishima 1988; van der Klis 1994). Their origin remains as one of the big mysteries of X-ray astronomy.

We have proposed a simple cellular-automaton model based on the notion of self-organized criticality (Bak, Tang \& Wiesenfeld 1988). In this model, we conjecture that the inner portion of the disk is composed of numerous reservoirs with finite capacities. By imposing the cellular-automaton 

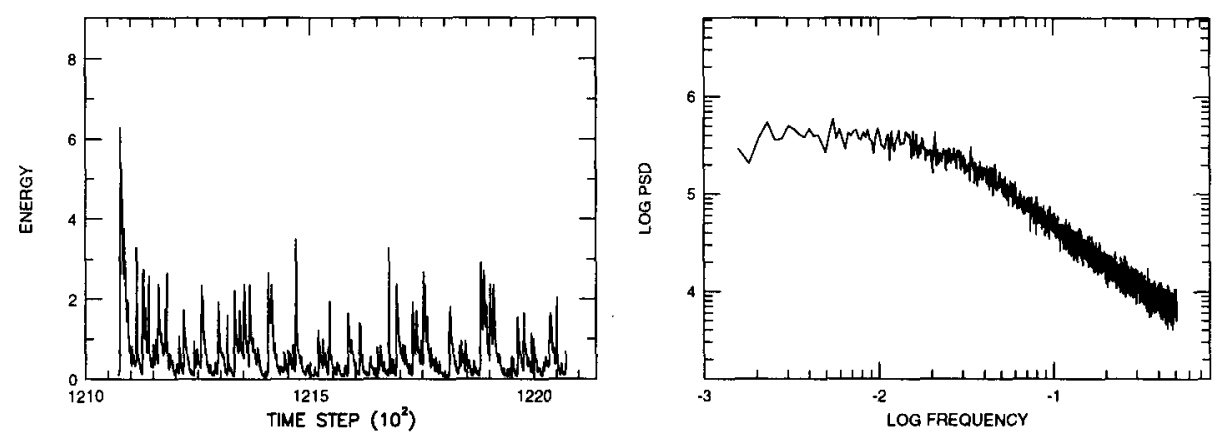

Figure 1. Typical light curves (left) and their PSD (right) produced by the Monte-Carlo simulations based on the SOC model. We set $m^{\prime}=m / 10$.

rule that mass accretion occurs when a mass density exceeds some critical value, we have succeeded in producing the basic features of X-ray fluctuations. In the following, we briefly outline the essence of our model (cf. Mineshige, Ouchi \& Nishimori 1994; Mineshige, Takeuchi \& Nishimori 1994).

\section{Cellular-automaton procedures}

(i) Divide the inner portions of an accretion disk into numerous small segments (or cells);

(ii) input a mass of $m$ into an outermost cell;

(iii) when a mass density exceeds some critical value, let three mass particles ( $3 \mathrm{~m})$ fall from that cell into the inner three adjacent cells (avalanche);

(iv) in addition to procedure (iii), let a mass of $m^{\prime}(\ll m)$ fall into the adjacent inner cell at each ring (gradual diffusion);

(v) calculate X-ray luminosities, assuming that a certain fraction of the released potential energy is dissipated via radiation;

(vi) repeat (i)-(v) more than $10^{5}$ times, plot light curves, calculate PSDs.

\section{SOC model and the X-ray observations}

The system then evolves to, and stays at, a self-organized critical state, exhibiting the following characteristics:

(i) aperiodic variations appear in the X-ray light curves (Fig. 1; left). Their PSDs show a $1 / f$-like decline at large frequencies (Fig. 1; right);

(ii) the peak intensities $(p)$ of individual shots are smoothly distributed over a wide range (Fig. 2). Roughly, the distribution is $D(p) \propto p^{-8}$ both in our SOC model with $m^{\prime}=m / 10$ and the observations. 

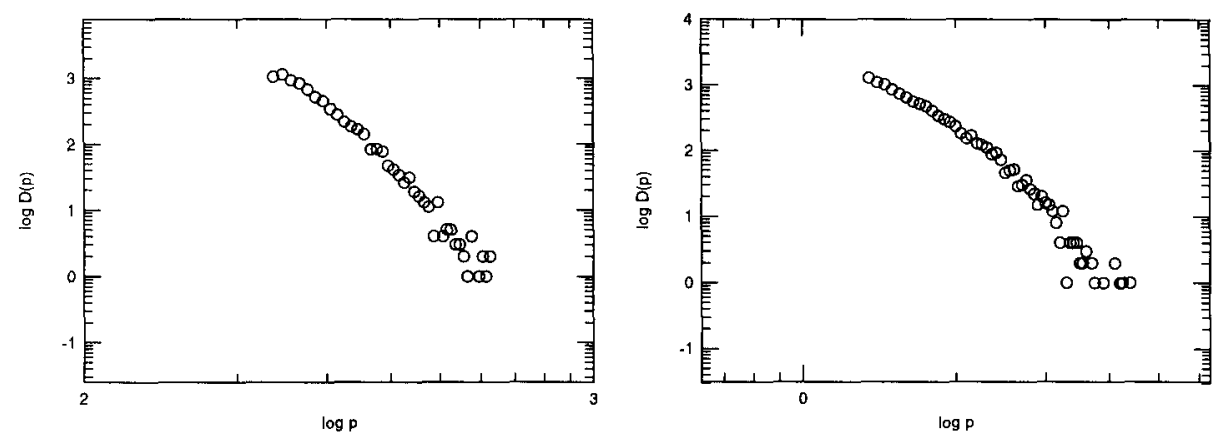

Figure 2. The observational (left) and theoretical (right) peak-intensity distribution of shots (from Negoro et al. 1995).

(iii) large shots tend to occur after an interval and this interval (waiting time) increases as $p$ increases. This is because once a large shot occurs, a long waiting time is needed to refill an accretion disk with gas until a next large event takes place.

These three characters appear both in our SOC model (Mineshige et al. 1994b) and in the Ginga observations of Cyg X-1 (Negoro et al. 1995).

\section{Discussion}

Through the present analysis, it has been established that X-ray shots of Cyg $\mathrm{X}-1$ occur according to a certain rule, although it looks as if they would randomly appear. What can we learn from this analysis about the structure of accretion flow onto compact objects?

First, the origin of the seed fluctuations is most likely to be magnetic activity, since magnetic fields tend to dissipate energy sporadically via reconnection, even if energy input is continuous. In fact, $\mathrm{Lu} \&$ Hamilton (1991) claimed to reproduce power-law occurrence rates of solar flares by applying the concept of SOC to the solar magnetic fields. In the conventional picture of solar-type accretion disks in which magnetic activities are only enhanced in the chromosphere-corona, however, large-amplitude fluctuations are not expected. We thus conjecture that the disk is of low- $\beta$, (where $\beta$ is the ratio of gas pressure to magnetic pressure) i.e., magnetic pressure dominated (cf. Shibata, Tajima \& Matsumoto 1990).

Second, there exist the so-called advection-dominated disks characterized by hot temperatures (and thus hard spectra), low radiation emissivity, and large infall velocity - sometimes comparable to the azimuthal velocity (Narayan \& Yi 1994; Abramowicz et al. 1995). Such disks are favored 


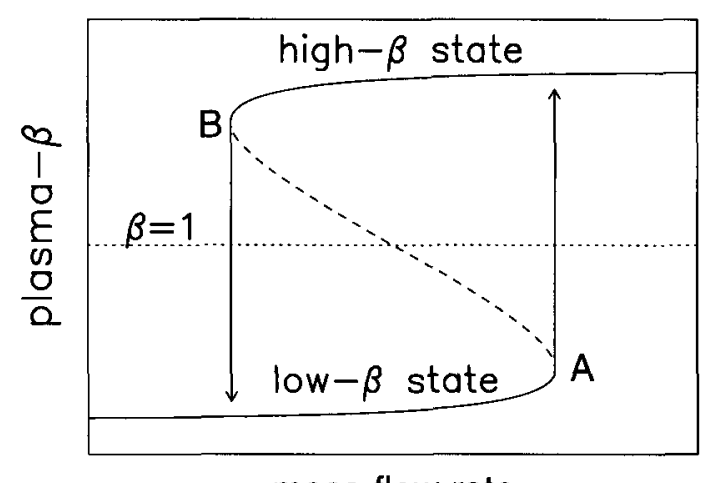

mass-flow rate

Figure 3. Hysteresis relation between the low- $\beta$, advection-dominated branch with enhanced variability (lower) and the high- $\beta$, standard branch with little variability (upper).

to produce fluctuations according to our model (Mineshige, Kusunose \& Matsumoto 1995), partly because then magnetic activity can be enhanced, and partly because mass flow then occurs both in the azimuthal and radial directions (a basic assumption of our model; see Sect. 2). The main energy release thus occurs by sporadic reconnection events, leading to magnetic eruptions like solar flares.

Finally, black-hole accretion disks seem to alternate between the optically thin, advection dominated state with enhanced variability and the optically thick, standard state with little variability (Fig. 3). This will explain hard spectra of transient black-hole candidates during the rise and late decay phase, and soft spectra in the early decay phase (Mineshige et al. 1995).

\section{References}

Abramowicz, M.A., Chen, X., Kato, S., et al., 1995, Ap. J., 438, L37

Bak, P., Tang, C., Wiesenfeld, K., 1988, Phys. Rev., A38, 364

Lu, E.T., Hamilton, R.J., 1991, Ap. J., 380, L89

Makishima, K., 1988, in "Physics of Neutron Stars and Black Holes" ed. Y. Tanaka, Universal Academy Press, Dordrecht, p175

Mineshige, S., Kusunose, M., Matsumoto, R., 1995, Ap. J., 445, L43

Mineshige, S., Ouchi, N.B., Nishimori, H., 1994a, PASJ, 46, 97

Mineshige, S., Takeuchi, M., Nishimori, H., 1994b, Ap. J., 435, L125

Narayan, R., Yi, I., 1994, Ap. J., 428, L13

Negoro, H., Kitamoto, S., Takeuchi, M., Mineshige, S., 1995, Ap. J., 452, L49

Oda, M., 1977, Space Sci. Rev., 20, 757

Shibata, K., Tajima, T., Matsumoto, R., 1990, Ap. J., 350, 295

van der Klis, M., 1994, Ap. J. Supp., 92, 511 